OPEN ACCESS

Edited by:

Pierina Visciano,

University of Teramo, Italy

Reviewed by:

Antonio Valero,

Universidad de Córdoba, Spain

Miriam R. Garcia,

Consejo Superior de Investigaciones

Cientificas (CSIC), Spain

*Correspondence:

Deog-Hwan Oh

deoghwa@kangwon.ac.kr

Specialty section: This article was submitted to

Food Microbiology,

a section of the journal

Frontiers in Microbiology

Received: 03 March 2017

Accepted: 06 June 2017

Published: 22 June 2017

Citation:

Wang J, Koseki S, Chung M-J and Oh D-H (2017) A Novel Approach to Predict the Growth of Staphylococcus aureus on Rice Cake.

Front. Microbiol. 8:1140

doi: 10.3389/fmicb.2017.01140

\section{A Novel Approach to Predict the Growth of Staphylococcus aureus on Rice Cake}

\author{
Jun Wang ${ }^{1}$, Shige Koseki ${ }^{2}$, Mi-Ja Chung ${ }^{3}$ and Deog-Hwan Oh ${ }^{4 *}$ \\ ${ }^{1}$ College of Food Science and Engineering, Qingdao Agricultural University, Qingdao, China, ${ }^{2}$ Research Faculty of \\ Agriculture, Hokkaido University, Sapporo, Japan, ${ }^{3}$ Department of Food Science and Nutrition, College of Health, Welfare \\ and Education, Gwangju University, Gwangju, South Korea, ${ }^{4}$ Department of Food Science and Biotechnology, Institute of \\ Bioscience and Biotechnology, Kangwon National University, Chuncheon, South Korea
}

This study aimed to investigate the growth kinetics of Staphylococcus aureus on rice cake and to determine the shelf life based on the probability model of the increase in S. aureus contamination on rice cake. Secondary models were developed based on the growth parameters derived from the Baranyi model at constant temperatures $\left(15,25,35\right.$, and $\left.45^{\circ} \mathrm{C}\right)$. External validation was then conducted using additional data under experimental conditions not used in development of the models to verify the performance and reliability of the developed model through different goodness-of-fit indices. Furthermore, the growth of $S$. aureus on rice cake under dynamic temperature was obtained with the root mean square error (RMSE) of 0.218 and the $90.9 \%$ acceptable prediction rate. In addition, probability models of the 1-, 2-, 3-, and 4-log increases of S. aureus on rice cake were also developed from the data, which could provide the probability and the time to a certain log increase. The results of validation demonstrated that the developed predictive model and the obtained growth parameters could be used for evaluating the growth behavior of $S$. aureus on rice cake under different conditions, and qualified to supply sufficient information for microbiological risk assessment studies of $S$. aureus on rice cake in Korea.

Keywords: Staphylococcus aureus, predictive microbiology, rice cake, growth model, probability model

\section{INTRODUCTION}

Rice cake is one of the most popular Korean traditional foods. Many types of rice cake can be prepared via the use of different ingredients and different manufacturing processes. Gyungdan, a ball-shaped rice cake filled with delicious, sweet red bean paste and rolled in sesame seeds, is the most common variety of "tteok." The major ingredients of rice cakes are rice flour and water, which causes rapid starch retrogradation during storage (Riva et al., 2000; Wu et al., 2009). Korean traditional rice cakes are generally packaged with linear low-density polyethylene after steaming and cooling at room temperature, and then distributed throughout markets (Lee et al., 2011). Oh et al. (2007) reported that $19.3 \%$ of the rice cakes with filling in Korea were contaminated with Staphylococcus aureus. The most important bacteria causing food poisoning in Korea are Salmonella spp., Vibrio spp., and S. aureus. These bacteria account for $85-90 \%$ of the outbreaks and cases of illness in Korea (Park et al., 2001). In addition, Salmonella spp., S. aureus, and Bacillus cereus are the major microbiological hazards of cereal grains and related products (FDA, 2003). 
In the past decades, foodborne illness has been a serious public health issue in both developed and developing countries. $S$. aureus is able to produce a variety of toxins and many disease syndromes, and many detection method of $S$. aureus were developed and reported (Dabrowski and Medrala, 2004; Zhao et al., 2014; Li et al., 2016). In the United States, total 17 foodborne outbreaks caused by Staphylococcus enterotoxin and 566 outbreak-associated illnesses were reported to the CDC in 2014 (CDC, 2016). In Korea, $22.76 \%$ of the 10,676 foodborne illnesses from 1981 to 1995 caused by bacteria were associated with S. aureus strains, while $10.8 \%$ of the 33,353 patients suffering from food poisoning from 2001 to 2005 had illness related to enterotoxins produced by $S$. aureus (Lee et al., 2001; Yoon et al., 2011). Risk related to S. aureus and staphylococcal enterotoxins in fluid milk were estimated and demonstrated from the consumption of milk (Ding et al., 2016). It is necessary to estimate the effects of environmental factors on the growth and survival of $S$. aureus on rice cake, as it is the most popular traditional food in Korea.

The methodologies of primary and secondary modeling approaches and validation indices are based on well-established in many publications. But the ways to develop the growth probability model and the kinetic models under fluctuating temperature indicating the different log-increase probability at different temperatures and including as a dependent variable in the logistic models, respectively, are rarely reported. The objective of the present study was to develop a novel approach to predict the growth kinetics of $S$. aureus on rice cake under various environmental conditions. The Baranyi and Roberts model and Ratkowsky square root equation were selected to develop the primary and secondary models to evaluate the growth of $S$. aureus on rice cake under $15,25,35$, and $45^{\circ} \mathrm{C}$. In addition, the kinetic models under fluctuating temperature for the growth of $S$. aureus on rice cake was developed along with a probability model of 1-, 2-, 3-, and 4-log increase were also developed based on the $S$. aureus growth data on rice cakes to identify the shelf life.

\section{MATERIALS AND METHODS}

\section{Preparation of Strains}

Staphylococcus aureus strains ATCC 12598, ATCC 25923, and ATCC 12600, obtained from the department of Food Science, University of Georgia, were used in this study. All strains were maintained at $-70^{\circ} \mathrm{C}$ in tryptic soy broth (TSB, Difco, Sparks, $\mathrm{MD}$, USA) containing $0.6 \%$ yeast extract (YE, Difco, USA) and $20 \%$ glycerol. For experimental purposes, the stock cultures of the strains were activated in tryptic soy broth (TSB; Difco, Detroit, $\mathrm{MI}, \mathrm{USA}$ ) at $35^{\circ} \mathrm{C}$ for $24 \mathrm{~h}$ in order to reach to the stationary phase. The bacteria were harvested by centrifugation $(3,000 \times \mathrm{g})$ at $4^{\circ} \mathrm{C}$ for $10 \mathrm{~min}$, and then washed twice using $0.1 \%(\mathrm{w} / \mathrm{v})$ sterile peptone water (PW; Difco, USA). The bacterial suspensions were mixed at equal concentrations to obtain a mixture with the final population level of approximately $5 \log \mathrm{cfu} / \mathrm{mL}$.

\section{Preparation and Inoculation of Samples}

Gyungdan, a ball-shaped rice cake filled with sweet red bean paste and rolled in sesame seeds, were purchased from a local supermarket in Chuncheon, Korea. The $\mathrm{pH}$ and water activity of rice cake was approximately 6.14 and 0.975 , respectively. The samples were cut in half using a sterile knife and placed on sterile aluminum foil in a lamella flow hood. The rice cakes were divided into $10 \mathrm{~g}$ samples with a balance to prepare for inoculation. For inoculation, $0.1 \mathrm{~mL}$ of the mixed culture (5 log $\mathrm{cfu} / \mathrm{mL}$ ) was applied to the samples by depositing droplets with a micropipettor. This procedure resulted in an initial pathogen inocula level of approximately $3 \log \mathrm{cfu} / \mathrm{g}$.

\section{Growth Experiments of S. aureus}

The inoculated samples were packaged with polyethylene and transferred into the growth chamber (BF-600GC, BioFree, Seoul, Korea). Uninoculated samples were used as a control. For the purpose of model development, the inoculated samples were stored at $5,15,25,35$, and $45^{\circ} \mathrm{C}$ at two different levels (50 and $80 \%$ ) of relative humidity until they reached the stationary phase. As for model validation, the experiments were conducted at 10, 20,30 , and $40^{\circ} \mathrm{C}$ within the region of interpolation of the model. Sampling was generally carried out for enumeration based on the designed intervals, depending on the different incubation temperatures. Two samples were tested at each time interval. The growth studies were conducted in triplicate for each combination of conditions. Experimental period was set approximately $25 \mathrm{~h}$ at 35 and $45^{\circ} \mathrm{C}, 30 \mathrm{~h}$ at 30 and $40^{\circ} \mathrm{C}$, and approximately $50 \mathrm{~h}$ at 20 and $25^{\circ} \mathrm{C}$. However, longer periods were selected for the experiment at 5,10 , and $15^{\circ} \mathrm{C}$.

\section{Enumeration of $S$. aureus}

At each sampling time, the $10 \mathrm{~g}$ rice cake samples to be tested were taken from the growth chamber and put into a sterile 400$\mathrm{mL}$ stomaching bag with a filter (Nasco Whirl-Pak, Janesville, WI, USA) then mixed with $90 \mathrm{~mL}$ of $0.1 \%$ sterilized PW. Samples were then pummeled in a Seward Stomacher (400 Circulator, Seward, London, UK) at $200 \mathrm{rpm}$ for $2 \mathrm{~min}$. After homogenization, $1 \mathrm{~mL}$ of the sample suspension containing $S$. aureus was added into $9 \mathrm{~mL} 0.1 \%$ sterilized $\mathrm{PW}$, and serial 10 -fold dilutions were performed using $0.1 \%$ sterilized PW. Subsequently, $0.1 \mathrm{~mL}$ of the obtained samples or dilutions was plated in duplicate on Baird-Parker Agar (Difco Co., USA). After incubation of the plates at $35^{\circ} \mathrm{C}$ for $24 \mathrm{~h}$, colonies were counted and the data were expressed as a logarithm of the population $(\log \mathrm{cfu} / \mathrm{g})$. Means of cell populations from each treatment were calculated from three replications.

\section{Kinetic Growth Model Development Primary Modeling}

Experimental data of $S$. aureus growth on the rice cake samples were collected for each designed trial with the different combinations of temperature and relative humidity. The specific growth rate $(\mu, 1 / \mathrm{h})$ and lag time $(\mathrm{LT}, \mathrm{h})$ for each growth curve were generated by fitting the raw data into the Baranyi and Roberts model (Baranyi and Roberts, 1994). Analysis was carried out with $\mathrm{R}$ software (A language and environment for statistical computing. R Foundation for Statistical Computing, Vienna, Austria. ISBN 3-900051-07-0, URL http://www.R-project.org/). 
An R-software package nlstools was used to fit the Baranyi and Roberts model.

\section{Secondary Modeling}

The Ratkowsky's square root model (Equation 1; Ratkowsky et al., 1982; Oscar, 2002) was used to develop secondary models for the $\mu$, obtained from different primary models by non-linear regression using the Origin 8.5 software package (OriginLab corporation, Northampton, MA 01060, USA) based upon the generated growth parameters.

$$
\sqrt{\mu}=a\left(T-T_{\min }\right)
$$

where $\mu$ is specific growth rate $(1 / \mathrm{h}), T$ is the temperature $\left({ }^{\circ} \mathrm{C}\right)$, $T_{\text {min }}$ is the minimum temperature $\left({ }^{\circ} \mathrm{C}\right)$ required for growth, and $a$ is the regression coefficient.

\section{Differential Secondary Modeling}

Staphylococcus aureus growth under fluctuating temperature.

In order to simulate the growth of $S$. aureus on rice cake, the Baranyi and Roberts (1994) was implemented as follows:

$$
\begin{aligned}
\frac{d q}{d t} & =\mu q, q(0)=q_{0} \\
\frac{d N}{d t} & =\frac{q}{1+q} \mu\left(1-\frac{N}{N_{\max }}\right) N, N(0)=N_{0}
\end{aligned}
$$

where $N$ denotes the bacterial cell concentration $(\mathrm{CFU} / \mathrm{g})$ at time $t, q$ is a dimensionless quantity related to the physiological state of the cells, $\mu$ is the maximum specific growth rate $(1 / \mathrm{h})$, and $N_{\text {max }}$ represents the maximum population density of the bacteria (CFU/g). The $q_{0}$ and $N_{0}$ represents the initial value of the $q$ and $N$, respectively. For the initial value of $q_{0}$ which is a measure of the initial physiological state of the cells, a geometric mean value for the physiological state parameter $\alpha_{0}$ was estimated from the constant temperature experimental data. It should be noted that the relationship between lag time $(\lambda)$ and $\alpha_{0}$ could be shown as follows:

$$
\mu \times \mathrm{LT}=\ln \left(1+\frac{1}{q_{0}}\right)=-\ln \left(\alpha_{0}\right)
$$

The models for $\mu$ (Equation 1) along with $N_{\max }$ and $q_{0}$ were substituted into Equations $(2,3)$, and the temperature allowed to be dependent on time. The system was solved numerically by the fourth-order Runge-Kutta method as a means of obtaining predictions of bacterial concentration using statistical software $\mathrm{R}$ (deSolve package). The temperature profile was recorded continuously using a data logger with an interval of $10 \mathrm{~s}$ and set to stimulate real life conditions during storage and transportation.

\section{Growth Probability Model Development}

For each growth probability model development, each sampling point was scored with values of 0 and 1 to indicate whether or not to obtain 1-, 2-, 3-, and 4-log increases of S. aureus growth, respectively. The probability of a 1-, 2-, 3-, and 4$\log$ increases of $S$. aureus from the growth data on rice cakes was collected and fitted to a logistic regression model using $\mathrm{R}$ software (A language and environment for statistical computing.
R Foundation for Statistical Computing, Vienna, Austria. ISBN 3 900051-07-0, URL http://www.R-project.org/). The analyses were made with the $g \operatorname{lm}()$ function that fits generalized linear models in R-software package STATS. The initial model for fitting had the following form after a minor modification according to the approach described by Ratkowsky and Ross (1995), Koseki et al. (2009), and Agresti (2007):

$$
\operatorname{Logit}(P)=\alpha_{0}+\alpha_{1} \text { Temp }+\alpha_{2} \cdot \ln (\text { Time })
$$

where $P$ is the probability of an arbitrary log-increase based on $S$. aureus growth, Logit $(P)=\ln \left(\frac{P}{1-P}\right), \alpha_{0}-\alpha_{2}$ are the coefficients to be estimated, Temp is the storage temperature, and Time is the time at which the increase of $S$. aureus growth reached the set values.

To evaluate the goodness-of-fit the developed model, the maximum rescaled $R$-square statistic, the Hosmer-Lemeshow goodness-of-fit statistic, and the receiver operating characteristic (ROC) curve were used (Agresti, 2007). The maximum rescaled $R^{2}$ for use with binomial error was proposed as a generalization of the coefficient of determination $R$ that is commonly used in regression applications involving normally distributed error (Nagelkerke, 1991; Tienungoon et al., 2000). The HosmerLemeshow goodness-of-fit statistic, which involves grouping objects into a contingency table and calculating a Pearson chisquare statistic, was proposed as a means of estimating goodness of fit (Tienungoon et al., 2000). Small values of the statistic (large $P$-values) indicate a good fit of the model to the data. The area under the ROC curve, $c$, is a measure of discrimination, obtained from a plot of sensitivity, i.e., the proportion of observed events that were correctly predicted to be events, against the complement of specificity, i.e., the proportion of nonevents that were correctly predicted to be nonevents. The closer the value of $c$ is to 1 , the greater is the discrimination. In epidemiological studies, a $c$ value of $>0.7$ is considered acceptable discrimination, a $c$ value of $>0.8$ as good discrimination, and a $c$ value of $>0.9$ as excellent discrimination (Lemeshow and Jean-Roger, 1994).

\section{Validation of the Predictive Models}

The performance and reliability of the developed models should be validated before actual application. The validation step was conducted using experimental data which was not used for model development. In the validation step, several statistical indices, such as bias factor $\left(B_{f}\right.$, Equation 6$)$, accuracy factor $\left(A_{f}\right.$, Equation 7 ), the root mean square error (RMSE, Equation 8; McKellar and Lu, 2004) and \%standard error of prediction (\%SEP, Equation 9; Garcia-Gimeno et al., 2005) were employed as follows:

$$
\begin{aligned}
B_{f} & =10\left(\sum_{i=1}^{n} \log \left(\mu_{\text {observed }} / \mu_{\text {predicted }}\right) / n\right) \\
A_{f} & =10\left(\sum_{i=1}^{n}\left|\log \left(\mu_{\text {predicted }} / \mu_{\text {observed }}\right)\right| / n\right) \\
R M S E & =\sqrt{\frac{\sum\left(\mu_{\text {observed }}-\mu_{\text {predicted }}\right)^{2}}{n}} \sqrt{\frac{\sum\left(\mu_{\text {observed }}-\mu_{\text {predicted }}\right)^{2}}{n}}(9) \\
\% \text { SEP } & =\frac{100}{\text { Average }\left(\mu_{\text {observed }}\right)}
\end{aligned}
$$


where $n$ is the number of observations, $\mu_{\text {observed }}$ is the observed value, and $\mu_{\text {predicted }}$ is the predicted value.

Differential secondary model performance was evaluated using the RMSE. In addition, the acceptable prediction zone method was used for evaluation of the model performance (Oscar, 2005). The prediction errors or relative errors (REs) for the individual fitted cases were calculated according to the following equation:

$$
\mathrm{RE}=\frac{\text { Predicted }- \text { Observed }}{\text { Predicted }}
$$

The model gives fail-safe predictions when the RE is $<0$, while fail-dangerous predictions at $\mathrm{RE}$ values $>0$. The proportion of $\mathrm{RE}$ (pRE) in the acceptable prediction zone of $\mathrm{RE}-0.6$ to 0.3 , wherein the proportion of $\geq 0.70$ indicated an acceptable model, was used to evaluate the performance of the obtained model.

\section{Statistical Analysis}

All the data from the three replicates were expressed as log colony forming units per gram (log CFU/g) and used together for model development. Statistical analysis was performed using IBM SPSS statistics 20 (IBM Corporation, New York, USA). Data were expressed as means \pm standard deviation (SD). All data were analyzed using Analysis of variance (ANOVA). Statistical significance of growth parameters was analyzed by $t$-test at a significance level of 0.05 . Values of validation indices were calculated in a Microsoft Excel spreadsheet.

\section{RESULTS AND DISCUSSION}

\section{Growth of S. aureus on Rice Cake at Different Temperatures}

The experimental data of $S$. aureus on rice at all the designed temperatures for model development and validation were collected and presented in Figures 1, 2, respectively. Experimental observations revealed $S$. aureus to be undetectable on the uninoculated rice cake samples (control). S. aureus did not grow well on the rice cake stored at 5 and $10^{\circ} \mathrm{C}$ (Figure 3), and a decline in the $S$. aureus cells was observed at $5^{\circ} \mathrm{C}$. This was similar with previous studies which indicated $S$. aureus could grow at temperatures as low as $7^{\circ} \mathrm{C}$ (ICMSF, 1980). In contrast, no growth was observed at $10^{\circ} \mathrm{C}$ for the storage period of up to 7 days. The reason may be because the target samples were different food matrices, and Staphylococci are particularly sensitive to nutrient depletion. The data at these lower incubation temperatures $\left(5\right.$ and $10^{\circ} \mathrm{C}$ ) were not included in the development and validation of the model. The final cell populations of $S$. aureus at $15,25,35$, and $45^{\circ} \mathrm{C}$ were $7.45,7.55,7.44$, and $7.65 \log$ $\mathrm{CFU} / \mathrm{mL}$, respectively, indicating that higher temperatures led to higher final cell populations. Among them, the higher values at 15 and $25^{\circ} \mathrm{C}$ are due to the higher initial population levels. Similar results were demonstrated in our previous research, in which the temperature was more influential on pathogen growth on the food matrix than humidity (Wang and Oh, 2012; Wang et al., 2012).

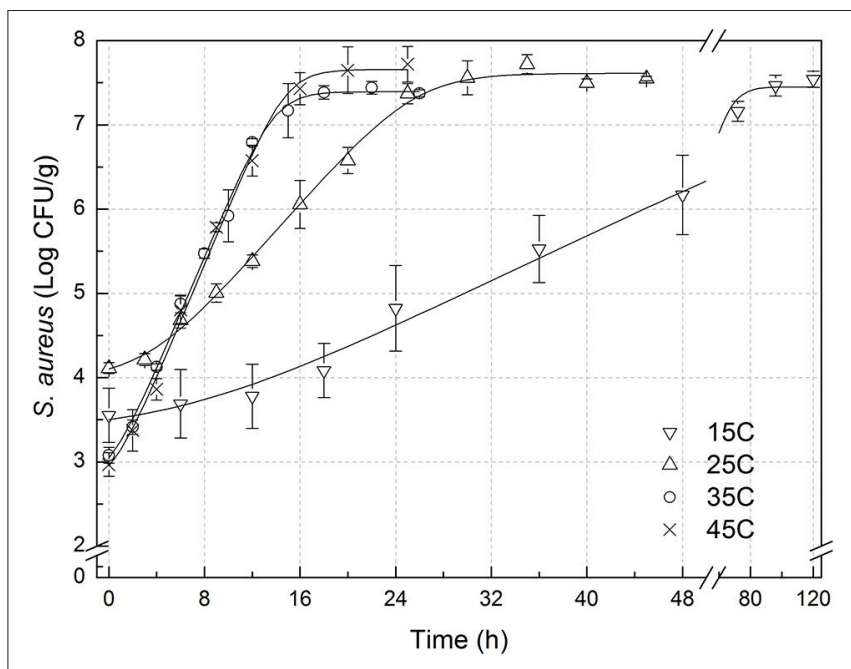

FIGURE 1 | Observed growth data and fitted primary models of Staphylococcus aureus on rice cake at $15,25,35$, and $45^{\circ} \mathrm{C}$.

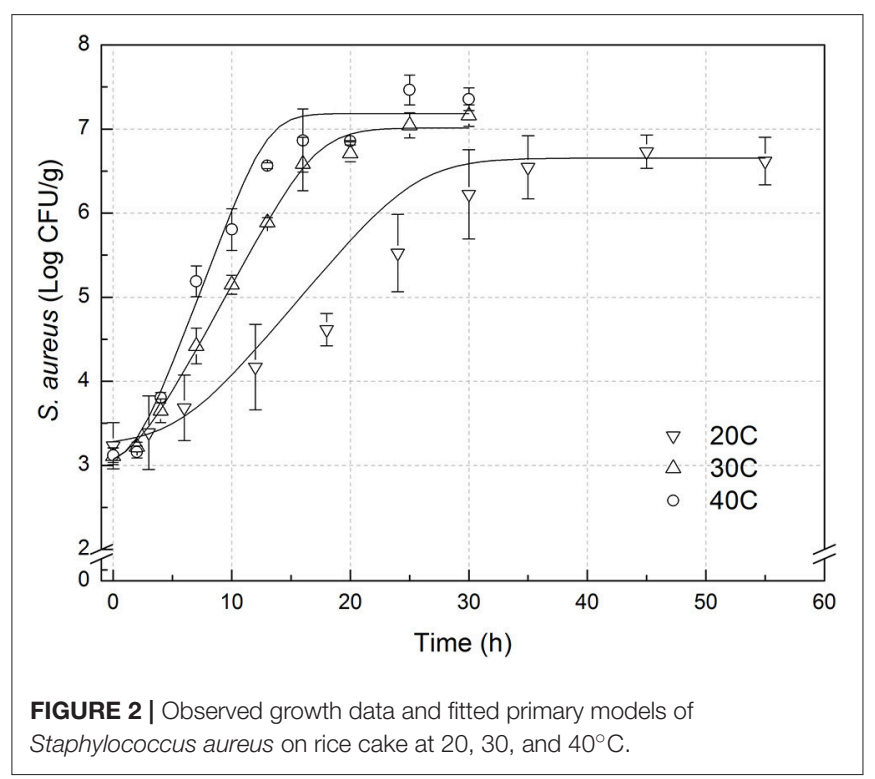

\section{Development of S. aureus Growth Models on Rice Cake Development of Primary Models}

In order to develop suitable primary models, which fit the growth data of $S$. aureus on rice cake, the growth curves of $S$. aureus at different environmental conditions were fitted into the Baranyi and Roberts model. The fitted curves at different temperatures were shown in Figures 1, 2. The results of analysis of the derived growth parameters including $\mu$ and LT indicated no significant differences between $\mu$ and LT values among different relative humidity $(p<0.05)$, regardless of the storage temperatures. Figures 1, 2 showed that lag phase could be only relevant for low temperatures. Therefore, the experimental data collected at the different relative humidity levels were combined to estimate 
specific growth rate of S. aureus on rice cake. The results of fitted parameters are summarized in Table 1.

\section{Development of Secondary Models}

Following the test of statistical significance of $\mu$ obtained from the primary models, only temperature was considered as a factor influencing the growth of $S$. aureus on rice cakes. The square root model, a special case of Belehradék's temperature function with the exponent of two, was suitable to describe the relationship between temperature and microbial growth rates (McMeekin et al., 2013). All $\mu$-values estimated from the primary models were employed to develop the secondary models using a square root model as follows:

$$
\sqrt{\mu}=0.0173^{*}(\mathrm{~T}+10.03)
$$

The fitted parameters of the Equation (11) are summarized in Table 2.

\section{Validation of Predictive Models}

In the present study, external validation was conducted using additional data under experimental conditions (Figure 2) which

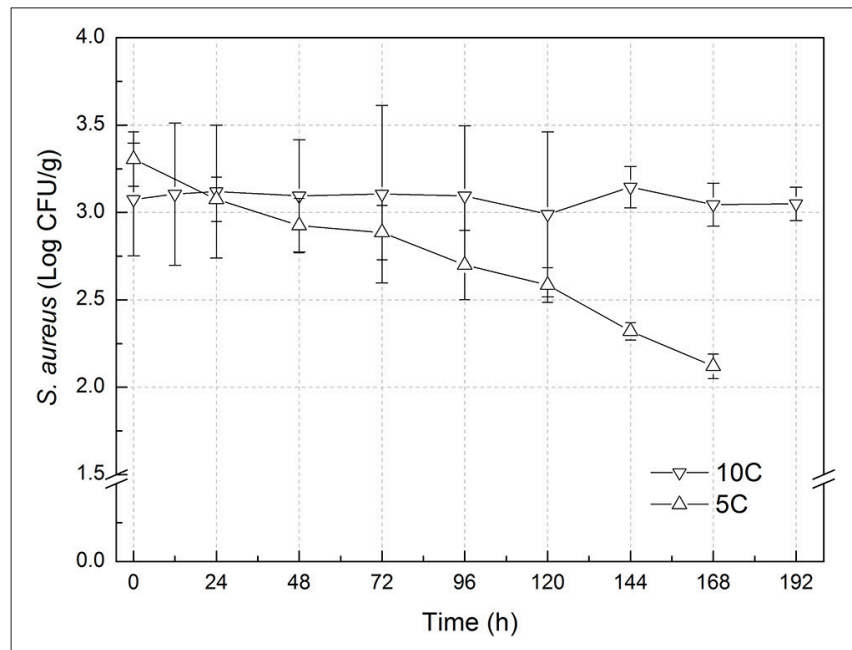

FIGURE 3 | Observed growth data of Staphylococcus aureus on rice cake at 5 and $10^{\circ} \mathrm{C}$. were not used for development of the models. Bias and accuracy factors are usually calculated for model validation. The $B_{f}$ value of the predictive model for $\mu$ was 1.131. Based on the criterion proposed by Ross (1999), the results indicated that the performance of developed models could be considered as acceptable. The acceptable range of an accuracy factor determined the number of environmental parameters in a kinetic model with $0.10-0.15$ units increased for each predictive variable (Ross et al., 2000). In this study, the acceptable range for $A_{f}$ should be $<1.15$ since just temperature was employed. The $A_{\mathrm{f}}$ value of the predictive model for $\mu$ was 1.131. The results indicated that the performance of developed model for $\mu$ could be considered as acceptable. RMSE was used to evaluate the performance of the predictive model. The lower the RMSE, the better goodness-of-fit the model shows (Wang et al., 2012). The RMSE value of the predictive model for $\mu$ was 0.086 . Previous works reported errors of the prediction of growth rate ranging from 0.27 to 0.30 (Sutherland et al., 1994; Olmez and Aran, 2005), which was greater than that for $\mu$ in the present study. The results indicated that the developed model showed a good quality of fit for the experimental data. The \%SEP has the advantage of being dimensionless, and calculation of the \%SEP for REs has the advantage of not being dependent on the magnitude of the measurements. The \%SEP value of the predictive model for $\mu$ on rice cake was $11.1 \%$. In several previous studies, it was reported that the \%SEP value for growth rate of Leuconostoc mesenteroides for external validation in aerobic conditions ranged from 14.37 to $22.88 \%$ (Garcia-Gimeno et al., 2005; Hervas-Martinez et al., 2006). Compared with the previously reported results, both values obtained herein proved the acceptable goodness of the proposed models.

TABLE 2 | Estimated Specific growth rate $\left(\mu_{\max }\right)$ of the secondary model for growth of Staphylococcus aureus on rice cake.

\begin{tabular}{llccc}
\hline & parameter & Value & L95CI $^{\mathbf{a}}$ & U95CI \\
\hline GR & a & 0.0173 & 0 & 0.034 \\
& Tmin & -10.03 & -51.5 & 31.4 \\
\hline
\end{tabular}

${ }^{a}$ Confidence intervals (95\%) of the estimates.

TABLE 1 | Growth parameters ( $\mu_{\max }$, specific growth rate and lag time) of Staphylococcus aureus on rice cake at different experimental conditions.

\begin{tabular}{|c|c|c|c|c|c|c|c|c|c|}
\hline Temperature $\left({ }^{\circ} \mathrm{C}\right)$ & $\mu \max (1 / h)$ & Std. Error & \multicolumn{2}{|c|}{$95 \% \mathrm{Cl}^{\mathrm{a}}$} & Lag time (h) & Std. Error & \multicolumn{2}{|c|}{$95 \% \mathrm{Cl}$} & $R^{2}$ \\
\hline $25^{\circ} \mathrm{C}$ & 0.372 & 0.013 & 0.346 & 0.398 & 3.850 & 0.624 & 2.627 & 5.073 & 0.989 \\
\hline $35^{\circ} \mathrm{C}$ & 0.771 & 0.024 & 0.724 & 0.818 & 0.865 & 0.330 & 0.218 & 1.512 & 0.989 \\
\hline $45^{\circ} \mathrm{C}$ & 0.779 & 0.029 & 0.722 & 0.836 & 0.923 & 0.398 & 0.143 & 1.703 & 0.987 \\
\hline $40^{\circ} \mathrm{C}$ & 0.858 & 0.084 & 0.694 & 1.023 & 1.993 & 0.866 & 0.296 & 3.690 & 0.959 \\
\hline
\end{tabular}

${ }^{a}$ Confidence intervals of the parameters. 


\section{S. aureus Growth on Rice Cake at Dynamic Temperatures}

To simulate growth of $S$. aureus on rice cake under real temperature profile, we calculated the number of $S$. aureus by using simultaneous differential equations (Equations 2, 3). The physiological state parameter $q_{0}$ was determined by average of the estimated fitted parameters of four iso-thermal growth curves. The $q_{0}$ of the tested growth curves of $S$. aureus was determined as $0.20 \pm 0.14$. Figure 4 demonstrated one of the representing results under fluctuating temperature condition. The RMSE for $S$. aureus growth on rice cake was 0.218 , while the acceptable prediction rate was $90.9 \%$. These indices exhibited acceptable

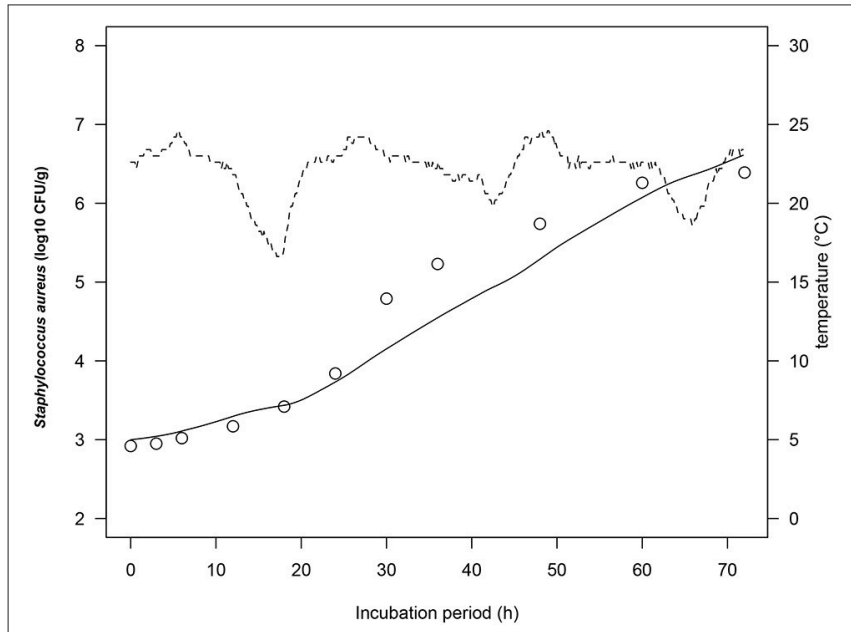

FIGURE 4 | Growth curves of Staphylococcus aureus on rice cake at dynamic temperatures (dashed lines) during the stimulated storage period. The solid line represents the prediction made from the developed model for $S$. aureus.

$\checkmark$ represents the observed values of $S$. aureus growth on rice cake. performance of the developed model for $S$. aureus growth on rice cake under the tested temperatures. In the present study, just one of the representing fluctuating temperature condition was monitored. The model was not tested for significant changes in temperature. Although the predictive capabilities of the model under dynamic temperature were not conducted, the calculation procedure would enable to correspond to real temperature history during distribution.

Fujikawa et al. (2004) developed a new logistic model for bacterial growth at dynamic temperatures using a numerical solution with the fourth-order Runge-Kutta method and demonstrated that the newly developed model could successfully predict Escherichia coli and Salmonellae growth curves for various patterns of the temperature history. Subsequently, $S$. aureus growth in sterilized milk were successfully predicted at various patterns of varying temperature using the newly developed logistic model, while its enterotoxin amounts predicted with the developed regression line for Staphylococcal enterotoxins were higher than the observed values (Fujikawa and Morozumi, 2006). In addition, comparison of the dynamic models based on the Baranyi model and modified logistic model could produce almost the same growth curves for varying temperature histories (Fujikawa and Morozumi, 2005).

\section{Development of Probabilistic Model}

In order to determine the effect of temperature on the growth of $S$. aureus on rice cake during the storage period, the probability of the time required to reach a certain level was determined using the logistic regression model described in Equation (4). The estimated parameters of the logistic regression models for 1-, 2- 3-, and 4-log increases of S. aureus expansion on rice cake are presented in Table 3. The information of goodnessof-fit of the model, such as $95 \%$ confidence intervals of the

TABLE 3 | Estimated parameters of the logistic regression for arbitrary growth increases of Staphylococcus aureus on rice cake.

\begin{tabular}{|c|c|c|c|c|c|c|c|c|}
\hline \multirow[t]{2}{*}{ Increases } & \multirow[t]{2}{*}{ Parameters } & \multirow[t]{2}{*}{ Estimates } & \multirow[t]{2}{*}{ Std. Error } & \multicolumn{2}{|c|}{$95 \% \mathrm{Cl}^{\mathrm{a}}$} & \multirow{2}{*}{$\begin{array}{l}\text { Maximum } \\
\text { rescaled } R^{2}\end{array}$} & \multirow[t]{2}{*}{$c$ (area under $\mathrm{ROC}^{\mathrm{b}}$ curve) } & \multirow{2}{*}{$\begin{array}{l}\text { Hosmer-lemeshow } \\
\text { (goodness of fit) }\end{array}$} \\
\hline & & & & Lower & Upper & & & \\
\hline \multirow[t]{3}{*}{$1-\log$} & $\alpha 0$ & -22.317 & 3.950 & -30.058 & -14.576 & 0.806 & 0.974 & 2.938 with $8 \mathrm{df}^{\mathrm{C}}(P=0.938)$ \\
\hline & $\alpha 1$ & 0.355 & 0.067 & 0.225 & 0.486 & & & \\
\hline & $\alpha 2$ & 5.835 & 1.002 & 3.872 & 7.799 & & & \\
\hline \multirow[t]{3}{*}{$2-\log$} & $\alpha 0$ & -32.48 & 5.542 & -43.342 & -21.618 & 0.831 & 0.981 & 4.492 with $8 \mathrm{df}(P=0.810)$ \\
\hline & $\alpha 1$ & 0.441 & 0.079 & 0.286 & 0.596 & & & \\
\hline & $\alpha 2$ & 7.667 & 1.288 & 5.143 & 10.191 & & & \\
\hline \multirow[t]{3}{*}{ 3-log } & $\alpha 0$ & -33.43 & 5.236 & -43.693 & -23.167 & 0.785 & 0.970 & 2.688 with $8 \mathrm{df}(P=0.952)$ \\
\hline & $\alpha 1$ & 0.435 & 0.072 & 0.294 & 0.576 & & & \\
\hline & $\alpha 2$ & 7.077 & 1.092 & 4.937 & 9.217 & & & \\
\hline \multirow[t]{3}{*}{$4-\log$} & $\alpha 0$ & -29.500 & 5.064 & -39.425 & -19.575 & 0.567 & 0.942 & 8.931 with $8 \mathrm{df}(P=0.348)$ \\
\hline & $\alpha 1$ & 0.400 & 0.070 & 0.263 & 0.537 & & & \\
\hline & $\alpha 2$ & 4.998 & 0.898 & 3.238 & 6.758 & & & \\
\hline
\end{tabular}

${ }^{a}$ Confidence intervals (95\%) of the estimates.

${ }^{b}$ Receiver operating curve.

${ }^{c}$ Degree of freedom. 

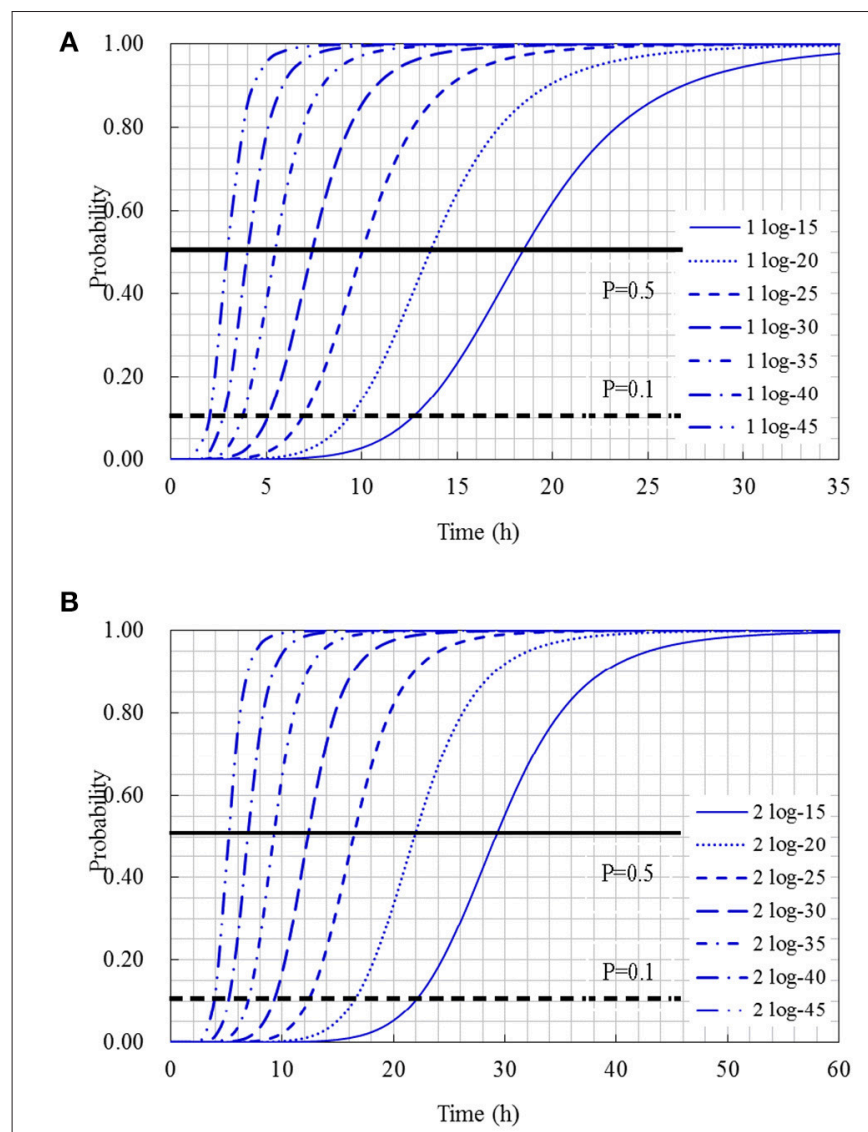

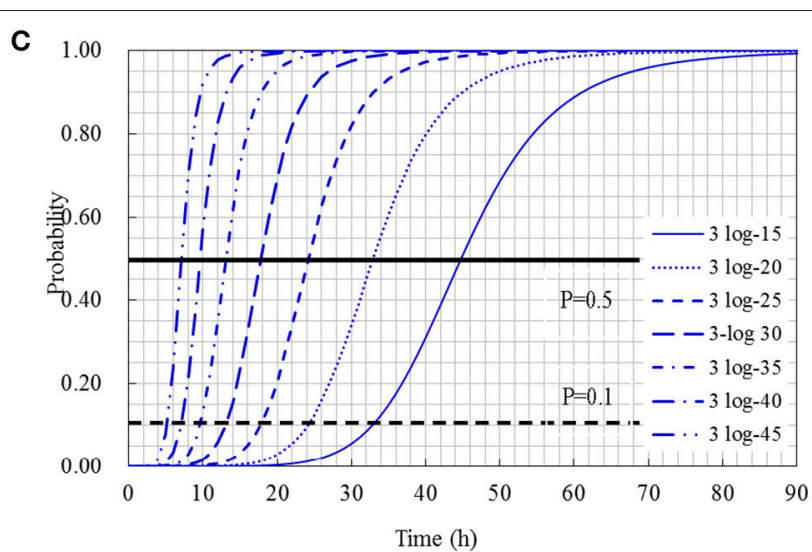

D

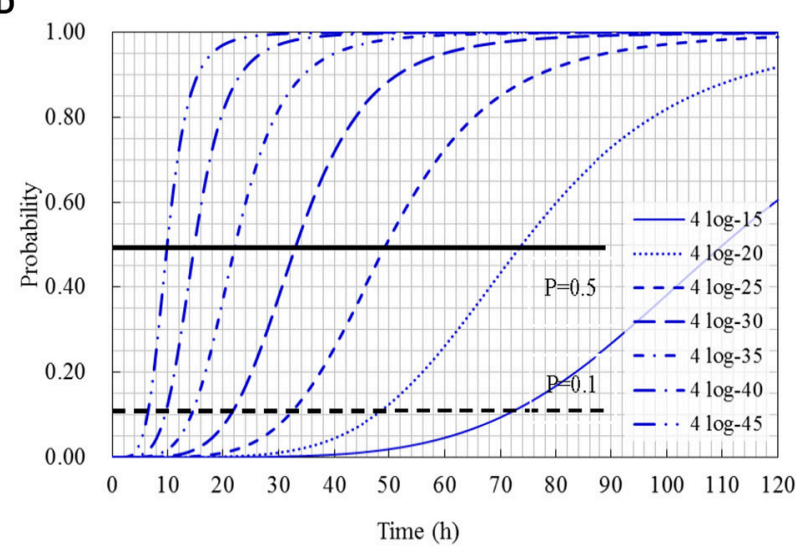

FIGURE 5 | Probability of a certain log increases of Staphylococcus aureus occurring at different temperatures: (A) 1-log increases; (B) 2-log increases; (C) 3-log increases; (D) $4-\log$ increases. Solid and dashed lines represent the probability $(p=0.5$ and $p=0.1)$ reaching to a certain log increases of $S$. aureus growth.

parameters, maximum rescaled $\mathrm{R}^{2}$, area under ROC curve, and Hosmer-Lemeshow goodness of fit are also listed in Table 3. According to the performance statistics, the derived parameters for the developed logistic regression models showed excellent discrimination for all the log increases.

The cumulative probability distributions predicted by the developed models listed in Table 3 are shown in Figure 5 for the 1-, 2-, 3-, and 4-log increases of S. aureus on rice cake, respectively. From the figure, the probability of the time required to reach a certain level of $S$. aureus contamination on the rice cakes at different temperatures could be determined. Meanwhile, the probability of the time required to reach different levels of $S$. aureus on rice cake at a certain temperature is presented in Figure 6.

Using the obtained model, the Logit $(\mathrm{P})$ values can be obtained, and the probabilities of $S$. aureus growth on rice cake can be estimated. Referred the reported limits on the growth of bacteria (Presser et al., 1998; Tienungoon et al., 2000; Le Marc et al., 2005; Hwang and Juneja, 2011) that $p \leq 0.1$ indicates an "unlikely to grow" or "no-growth" region, $p>0.5$ indicates a "likely to grow" or "growth" region, and p values between 0.1 and 0.5 indicate an "uncertainty" region, in the present study, the criterion of a certain $\log$ increases of $S$. aureus was set to be $P=0.5$ and $P=0.1$. That is to say, as for a certain level of logistic regression model, $P \leq 0.1$ indicates an "unlikely reach" region of a certain $\log$ increase, $P>0.5$ indicates a "reach" region, and $P$-values between 0.1 and 0.5 indicate a "likely reach" region (Figure 5). According to this criterion, we could derive an equation through transformation from the obtained logistic regression models shown in Table 3 to calculate the time to reach a certain log increase of $S$. aureus at different temperatures within the limits of the experimental design. The equations to calculate how long time to reach 1-, 2-, 3-, and 4-log increase of $S$. aureus were listed as following:

$$
\begin{array}{r}
t=\exp ((22.317-0.355 * \text { Temp }) / 5.835) \\
t=\exp ((32.48-0.441 * \text { Temp }) / 7.667) \\
t=\exp ((33.43-0.435 * \text { Temp }) / 7.077) \\
t=\exp ((29.50-0.400 * \text { Temp }) / 4.998)
\end{array}
$$

where Temp is the storage temperature, and $t$ is the time at which 1-, 2-, 3-, and 4-log increases of S. aureus reached, respectively.

Time and temperature abuse would lead to results of pathogenic bacteria growth and toxin formation. S. aureus concentrations of more than $10^{5} \mathrm{CFU} / \mathrm{mL}$ were unacceptable, since numbers of $S$. aureus higher than $10^{5}$ most often lead 

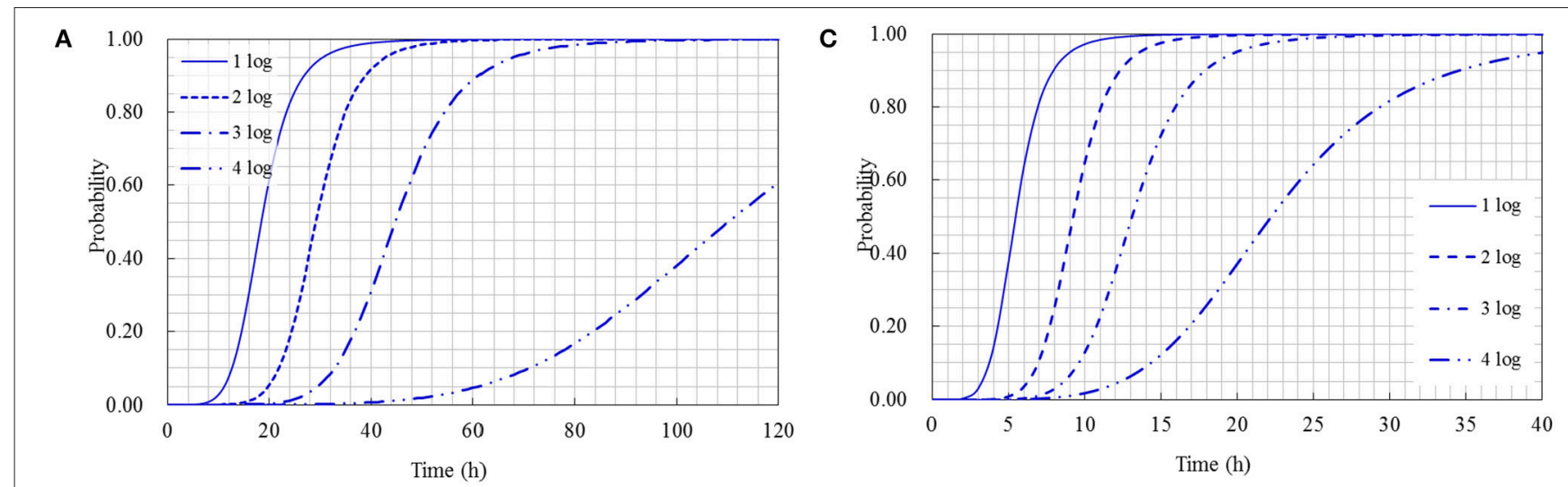

B

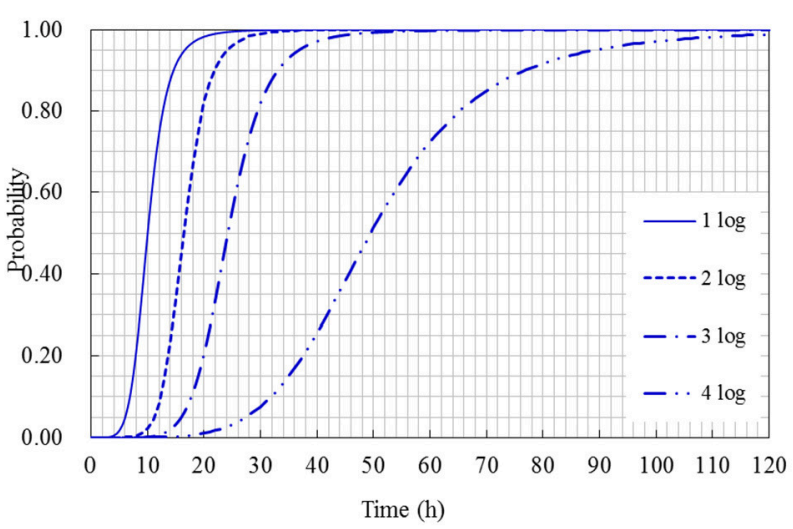

D

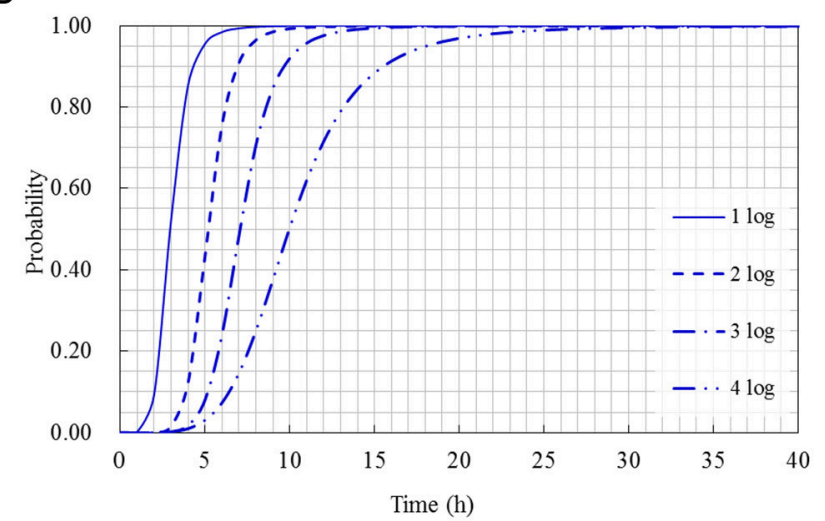

FIGURE 6 | Probability of different log increases of Staphylococcus aureus at a certain time at the following temperature: (A) $15^{\circ} \mathrm{C}$; (B) $25^{\circ} \mathrm{C}$; (C) $35^{\circ} \mathrm{C}$; (D) $45^{\circ} \mathrm{C}$.

to human illness (Food and Drug Administration, 2012). In addition, it is extremely likely to produce Staphylococcal enterotoxins under specific environmental conditions when the S. aureus level reaches $10^{5} \mathrm{CFU} / \mathrm{mL}$ (Heidinger et al., 2009). Fujikawa and Morozumi (2006) reported that temperature dependency of the rate constant of staphylococcal enterotoxin A (ng/mL/h) could be calculated as follows:

$$
\mathrm{p}_{\text {toxin }}=0.0376 * T-0.559
$$

where $\mathrm{p}_{\text {toxin }}$ is the production rate of staphylococcal enterotoxin $\mathrm{A}, \mathrm{T}$ is temperature. Based on Equation (16), enterotoxin production rate at each temperature would be estimated. According to Equation (14), it is easy to know the time to reach 3-log increase of $S$. aureus growth at different temperatures within the limits of the experimental design. As shown in Figure 5c, the area above the solid line was "reach" region, and the time to reach 3-log increase of $S$. aureus growth at 15, 25, 35 , and $45^{\circ} \mathrm{C}$ were $44.8,24.2,13.1$, and $7.1 \mathrm{~h}$, respectively. At the same time, the probability to reach 3-log increase could be obtained. Finally, the hazardous levels at each temperature could be evaluated considering the probability models and the enterotoxin production rate. If we could check the initial contamination level of rice cake, it is convenient to monitor the status of rice cake products. These logistic regression models could identify the shelf life of rice cake based on the initial levels of $S$. aureus contamination. From a food industry standpoint, to control the growth conditions of pathogens contributing to levels exceeding $10^{5}$ will be of key importance. Therefore, the developed probability models will be very useful for food safety management and microbiological risk assessment of $S$. aureus on rice cake in Korea.

\section{AUTHOR CONTRIBUTIONS}

The experiments were conceived and designed by JW, MC, and DO. Microbiological and data analyses were performed by JW and SK. The paper was written by JW, SK, and DO.

\section{ACKNOWLEDGMENTS}

This research was supported by a grant (10162KFDA995) from the Korea Food \& Drug Administration.

\section{SUPPLEMENTARY MATERIAL}

The Supplementary Material for this article can be found online at: http://journal.frontiersin.org/article/10.3389/fmicb. 2017.01140/full\#supplementary-material 


\section{REFERENCES}

Agresti, A. (2007). An Introduction to Categorical Data Analysis, 2nd Edn. Hoboken, NJ: John Wiley and Sons, Inc.

Baranyi, J., and Roberts, T. A. (1994). A dynamic approach to predicting bacterial growth in food. Int. J. Food Microbiol. 23, 277-294. doi: 10.1016/0168-1605(94)90157-0

CDC (2016). Surveillance for Foodborne Disease Outbreaks, United States, 2014, Annual Report. Atlanta: US Department of Health and Human Services.

Dabrowski, W. M., and Medrala, D. (2004). "Bacterial toxins," in Toxins in Food, eds W. M. Dabrowski and Z. E. Sikorski (Boca Raton, FL: CRC Press, LLC, US), 187-210.

Ding, T. Yu, Y. Y., Schaffner, D. W., Chen, S. G., Ye, Q. U., and Liu, D.-H. (2016). Farm to consumption risk assessment for Staphylococcus aureus and staphylococcal enterotoxins in fluid milk in China. Food Control 59, 636-643. doi: 10.1016/j.foodcont.2015.06.049

FDA. (2003). Analysis of microbial hazards related to time/temperature control of foods for safety. Compr. Rev. Food Sci. Food Safety 2, 33-41. doi: 10.1111/j.1541-4337.2003.tb00049.x

Food and Drug Administration (2012). "Staphylococcus aureus," Bad Bug Book, Foodborne Pathogenic Microorganisms and Natural Toxins. 2nd Edn. (FDA), 80-84. Available online at: https://www.fda.gov/downloads/Food/ FoodborneIllnessContaminants/UCM297627.pdf

Fujikawa, H., Kai, A., and Morozumi, S. (2004). A new logistic model for Escherichia coli growth at constant and dynamic temperatures. Food Microbiol. 21, 501-509. doi: 10.1016/j.fm.2004.01.007

Fujikawa, H., and Morozumi, S. (2005). Modeling surface growth of Escherichia coli on agar plates. Appl. Environ. Microbiol. 71, 7920-7926. doi: 10.1128/AEM.71.12.7920-7926.2005

Fujikawa, H., and Morozumi, S. (2006). Modeling Staphylococcus aureus growth and enterotoxin production in milk. Food Microbiol. 23, 260-267. doi: 10.1016/j.fm.2005.04.005

Garcia-Gimeno, R. M., Hervas-Martinez, C., Rodriguez-Perez, R., and ZureraCosano, G. (2005). Modelling the growth of Leuconostoc mesenteroides by artificial neural networks. Int. J. Food Microbiol. 105, 317-332. doi: $10.1016 /$ j.ijfoodmicro.2005.04.013

Heidinger, J. C., Winter, C., K., and Cullor, J., S. (2009). Quantitative microbial risk assessment for Staphylococcus aureus and Staphylococcus enterotoxin A in raw milk. J. Food Prot. 72, 1641-1653. doi: 10.4315/0362-028X-72. 8.1641

Hervas-Martinez, C., Garcia-Gimeno, R. M., Martinez-Estudillo, A. C., MartinezEstudillo, F. J., and Zurera-Cosano, G. (2006). Improving microbial growth prediction by product unit neural networks. J. Food Sci. 71, M31-M38. doi: 10.1111/j.1365-2621.2006.tb08904.x

Hwang, C. A., and Juneja, V. (2011). Effects of salt, sodium pyrophosphate, and sodium lactate on the probability of growth of Escherichia coli O157:H7 in ground beef. J. Food Prot. 74, 622-626. doi: 10.4315/0362-028X.JFP10-325

ICMSF (1980). Microbial Ecology of Foods. Vol. 1, Factors Affecting Life and Death of Microorganisms. Orlando, FL: International Commission on Microbiological Specification for Foods; Academic Press.

Koseki, S., Matsubara, M., and Yamamoto, K. (2009). Prediction of a required $\log$ reduction with probability for enterobacter sakazakii during high-pressure processing, using a survival/death interface model. Appl. Environ. Microb. 75, 1885-1891. doi: 10.1128/AEM.02283-08

Lee, K. A., Kim, K. T., and Paik, H. D. (2011). Physicochemical, microbial, and sensory evaluation of cook-chilled Korean traditional rice cake (Backseolgi) during storage via various packaging methods. Food Sci. Biotechnol. 20, 1069-1074. doi: 10.1007/s10068-0110145-Z

Lee, W. C., Lee, M. J., Kim, J. S., and Park, S. Y. (2001). Foodborne illness outbreaks in Korea and Japan studied retrospectively. J. Food Prot. 64, 899-902. doi: 10.4315/0362-028X-64.6.899

Le Marc, Y., Pin, C., and Baranyi, J. (2005). Methods to determine the growth domain in a multidimensional environmental space. Int. J. Food Microbiol. 100, 3-12. doi: 10.1016/j.ijfoodmicro.2004.10.003
Lemeshow, S., and Jean-Roger, L. (1994). Modeling the severity of illness of icu patients: a systems update. JAMA 272, 1049-1055. doi: 10.1001/jama.1994.03520130087038

Li, J., Ahn, J., Liu, D., Chen, S., Ye, X., and Ding, T. (2016). Evaluation of ultrasound-induced damage to Escherichia coli and Staphylococcus aureus by flow cytometry and transmission electron microscopy. Appl. Environ. Microbiol. 82, 1828-1837. doi: 10.1128/AEM.03 080-15

McKellar, R. C., and Lu, X. (2004). Modeling Microbial Responses in Food. Boca Raton, FL: CRC Press.

McMeekin, T., Olley, J., Ratkowsky, D., Corkrey, R., and Ross, T. (2013). Predictive microbiology theory and application: Is it all about rates? Food Control 29, 290-299. doi: 10.1016/j.foodcont.2012.06.001

Nagelkerke, N. (1991). A note on a general definition of the coefficient of determination. Biometrika 78, 691-692. doi: 10.1093/biomet/78. 3.691

Oh, S. K., Lee, N., Cho, Y. S., Shin, D. B., Choi, S. Y., and Koo, M. (2007). Occurrence of toxigenic Staphylococcus aureus in ready-to-eat food in Korea. J Food Protect 70, 1153-1158. doi: 10.4315/0362-028X-70. 5.1153

Olmez, H. K., and Aran, N. (2005). Modeling the growth kinetics of Bacillus cereus as a function of temperature, $\mathrm{pH}$, sodium lactate and sodium chloride concentrations. Int. J. Food Microbiol. 98, 135-143. doi: 10.1016/j.ijfoodmicro.2004.05.018

Oscar, T. P. (2005). Validation of lag time and growth rate models for Salmonella typhimurium: acceptable prediction zone method. J. Food Sci. 70, M129-M137. doi: 10.1111/j.1365-2621.2005.tb07103.x

Oscar, T. P. (2002). Development and validation of a tertiary simulation model for predicting the potential growth of Salmonella typhimurium on cooked chicken. Int. J. Food Microbiol. 76, 177-190. doi: 10.1016/S0168-1605(02) 00025-9

Park, H. O., Kim, C. H., Woo, G. J., Park, S. H., Lee, D. H., and Park, K. H. (2001). Monitoring and trends analysis of food poisoning outbreaks occurred in recent years in Korea. J. Food Hyg. Saf. 16, 280-294.

Presser, K. A., Ross, T., and Ratkowsky, D. A. (1998). Modelling the growth limits (growth/no growth interface) of Escherichia coli as a function of temperature, $\mathrm{pH}$, lactic acid concentration, and water activity. Appl. Environ. Microbiol. 64, 1773-1779.

Ratkowsky, D. A., Olley, J., McMeekin, T. A., and Ball, A. (1982). Relationship between temperature and growth rate of bacterial cultures. J. Bacteriol. 149, 1-5.

Ratkowsky, D. A., and Ross, T. (1995). Modeling the bacterialgrowth go growth interface. Lett. Appl. Microbiol. 20, 29-33. doi: 10.1111/j.1472-765X.1995.tb00400.x

Riva, M., Fessas, D., and Schiraldi, A. (2000). Starch Retrogradation in Cooked Pasta and Rice. Cereal Chem. J. 77, 433-438. doi: 10.1094/CCHEM.2000.77.4.433

Ross, T. (1999). Predictive Food Microbiology Models in the Meat Industry (MSRC.003). Sydney, NSW: Meat and Livestock.

Ross, T., Dalgaard, P., and Tienungoon, S. (2000). Predictive modelling of the growth and survival of Listeria in fishery products. Int. J. Food Microbiol. 62, 231-245. doi: 10.1016/S0168-1605(00)00340-8

Sutherland, J. P., Bayliss, A. J., and Roberts, T. A. (1994). Predictive modelling of growth of Staphylococcus aureus: the effects of temperature, $\mathrm{pH}$ and sodium chloride. Int. J. Food Microbiol. 21, 217-236. doi: 10.1016/0168-1605(94)90029-9

Tienungoon, S., Ratkowsky, D. A., McMeekin, T. A., and Ross, T. (2000). Growth limits of Listeria monocytogenes as a function of temperature, $\mathrm{pH}, \mathrm{NaCl}$, and lactic acid. Appl. Environ. Microbiol. 66, 4979-4987. doi: 10.1128/AEM.66.11.4979-4987.2000

Wang, J., Membre, J. M., Ha, S. D., Bahk, G. J., Chung, M. S., and Oh, D. H. (2012). Modeling the combined effect of temperature and relative humidity on Escherichia coli O157:H7 on lettuce. Food Sci. Biotechnol. 21, 859-865. doi: 10.1007/s10068-012-0111-4

Wang, J., and Oh, D. H. (2012). Effect of temperature and relative humidity on growth behavior of Escherichia Coli O157:H7 ON spinach using response surface methodology. J. Food Saf. 32, 296-304. doi: $10.1111 /$ j.1745-4565.2012.00380.x 
Wu, Y., Chen, Z., Li, X., and Li, M. (2009). Effect of tea polyphenols on the retrogradation of rice starch. Food Res. Int. 42, 221-225. doi: 10.1016/j.foodres.2008.11.001

Yoon, S. K., Ghafoor, K., Kang, Y. S., Sohn, M. G., Hwang, I. G., and Park, J. (2011). Exposure assessment of Staphylococcus aureus in Kimbab, a ready-toeat Korean food. Food Sci. Biotechnol. 20, 23-28. doi: 10.1007/s10068-0110004-y

Zhao, X., Lin, C. W., Wang, J., and Oh, D. H. (2014). Advances in rapid detection methods for foodborne pathogens. J. Microbiol. Biotechnol. 24, 297-312. doi: $10.4014 /$ jmb.1310.10013
Conflict of Interest Statement: The authors declare that the research was conducted in the absence of any commercial or financial relationships that could be construed as a potential conflict of interest.

Copyright (c) 2017 Wang, Koseki, Chung and Oh. This is an open-access article distributed under the terms of the Creative Commons Attribution License (CC BY). The use, distribution or reproduction in other forums is permitted, provided the original author(s) or licensor are credited and that the original publication in this journal is cited, in accordance with accepted academic practice. No use, distribution or reproduction is permitted which does not comply with these terms. 